\title{
Review
}

\section{The rheostat in the membrane: BCL-2 family proteins and apoptosis}

\author{
N Volkmann ${ }^{1,4}$, FM Marassi ${ }^{2,4}$, DD Newmeyer ${ }^{3,4}$ and D Hanein ${ }^{\star, 1,4}$
}

Apoptosis, a mechanism for programmed cell death, has key roles in human health and disease. Many signals for cellular life and death are regulated by the BCL-2 family proteins and converge at mitochondria, where cell fate is ultimately decided. The BCL-2 family includes both pro-life (e.g. BCL-XL) and pro-death (e.g. BAX, BAK) proteins. Previously, it was thought that a balance between these opposing proteins, like a simple 'rheostat', could control the sensitivity of cells to apoptotic stresses. Later, this rheostat concept had to be extended, when it became clear that BCL-2 family proteins regulate each other through a complex network of bimolecular interactions, some transient and some relatively stable. Now, studies have shown that the apoptotic circuitry is even more sophisticated, in that BCL-2 family interactions are spatially dynamic, even in nonapoptotic cells. For example, BAX and BCL-XL can shuttle between the cytoplasm and the mitochondrial outer membrane (MOM). Upstream signaling pathways can regulate the cytoplasmic-MOM equilibrium of BAX and thereby adjust the sensitivity of cells to apoptotic stimuli. Thus, we can view the MOM as the central locale of a dynamic life-death rheostat. BAX invariably forms extensive homo-oligomers after activation in membranes. However, recent studies, showing that activated BAX monomers determine the kinetics of MOM permeabilization (MOMP), perturb the lipid bilayer and form nanometer size pores, pose questions about the role of the oligomerization. Other lingering questions concern the molecular mechanisms of BAX redistribution between MOM and cytoplasm and the details of BAX/BAK-membrane assemblies. Future studies need to delineate how BCL-2 family proteins regulate MOMP, in concert with auxiliary MOM proteins, in a dynamic membrane environment. Technologies aimed at elucidating the structure and function of the full-length proteins in membranes are needed to illuminate some of these critical issues.

Cell Death and Differentiation (2014) 21, 206-215; doi:10.1038/cdd.2013.153; published online 25 October 2013

Facts

- BH3-only proteins (e.g. BID, BIM and PUMA) promote MOM integration of BAX/BAK, leading to MOMP, which typically commits the cell to death.

- The cytoprotective activity of BCL-XL is exerted through interactions with $\mathrm{BAX} / \mathrm{BAK}$ and $\mathrm{BH} 3$-only proteins.

- Non-activated BAX and BCL-XL cycle between cytosolic and MOM-associated states. In some cells, they can cause each other to retrotranslocate from the MOM back to the cytoplasm. However, in other situations, BCL-XL stabilizes BAX association with the MOM.

- MOM-resident proteins, both identified and unidentified, have been proposed to facilitate the MOM permeabilizing function of BAX.

\section{Open Questions}

- How do BAX and BAK permeabilize the MOM?

- How do the pro-survival family members, e.g. BCL-XL, protect against cell death?
- How do BCL-2 family proteins function together in the MOM?

- Which MOM proteins facilitate retrotranslocation and permeabilization and by what mechanisms?

\section{Recent Results}

- Membrane-inserted BAX monomers control the kinetics of permeabilization, induce the formation of lipidic pores and significantly distort phospholipid bilayers.

- BAX activation in the MOM triggers the assembly of a nonBAX 'catalyst' complex, which then facilitates BAX pore formation.

- Some of the initial conformational events in BAX and BAK activation have been identified by structural studies. BAX and BAK can both be activated directly by certain $\mathrm{BH}$-only proteins. For BAK, this activation involves $\mathrm{BH} 3$ domain binding to the 'canonical groove', but for BAX, activator $\mathrm{BH}$ domain binding occurs at a different surface.

${ }^{1}$ Bioinformatics and Systems Biology Program, Sanford Burnham Medical Research Institute, La Jolla, CA 92037, USA; ${ }^{2}$ Apoptosis and Cell Death Research Program, Sanford Burnham Medical Research Institute, La Jolla, CA 92037, USA and ${ }^{3}$ Division of Immune Regulation, La Jolla Institute for Allergy and Immunology, La Jolla, CA, USA

${ }^{*}$ Corresponding author: D Hanein, Bioinformatics and Systems Biology Program, Sanford Burnham Medical Research Institute, 10901 North Torrey Pine Rd., La Jolla, CA 92037, USA. Tel: +858 646 3134; Fax: +858 646 3172; E-mail: dorit@burnham.org

${ }^{4}$ All authors contributed equally to this review.

Keywords: BCL-XL; BAX; BID; mitochondria; structure; membrane

Abbreviations: MOM, mitochondrial outer membrane; MOMP, MOM permeabilization

Received 03.7.13; revised 22.8.13; accepted 17.9.13; Edited by C Borner; published online 25.10.2013 
- Survival signals can promote BAX retrotranslocation from the MOM to the cytoplasm (reducing the amount of BAX in the MOM); on the other hand, the loss of survival signals causes enhanced MOM accumulation of BAX and sensitizes cells to death signals.

\section{Apoptosis and BCL-2 Family Proteins}

Apoptosis is an active, intracellular cell death program that is common to nearly all higher eukaryotic cell types. ${ }^{1}$ As a counterbalance to cellular proliferation, this process helps to maintain cellular homeostasis. Consequently, its deregulation can seriously affect health. Abnormally suppressed apoptosis has key roles in many human diseases, including tumor development and tumor cell resistance to chemotherapy. The relevance of apoptosis to human health has propelled it to the forefront of biomedical research, with the hope that understanding its molecular mechanism will translate into new therapeutic approaches.

Early discoveries concerning the BCL-2 oncogene product, in particular that it suppresses cell death, counteracts the proapoptotic action of mitochondria and represents a family of anti- and pro-apoptotic proteins, ${ }^{2-6}$ provided some of the first insights into the molecular basis of programmed cell death and paved the way for subsequent studies that shape our current understanding of apoptosis. It is now well established that the BCL-2 family proteins are the central regulators of the mitochondrial cell-intrinsic apoptotic pathway. Although many kinds of stresses can induce apoptosis via diverse signaling pathways, these signals often converge at the mitochondrial outer membrane (MOM), where cell fate is ultimately decided. BCL-2 family proteins can have functions at non-mitochondrial sites (e.g. the endoplasmic reticulum). Nevertheless, perhaps the most prominent roles of these proteins are to promote or inhibit MOM permeabilization (MOMP), serving as a molecular 'rheostat' that regulates the mitochondrial pathway to apoptosis. ${ }^{7-21}$ MOMP induced by BAX and BAK allows cytotoxic proteins to escape from the mitochondrial intermembrane space into the cytoplasm, leading to caspase activation and apoptosis. MOMP also leads to progressive mitochondrial dysfunction, which causes energy depletion and cell death even when caspases are inactive. This means that cells can recover from MOMP only under exceptional circumstances. ${ }^{22-24}$ Even in cases where apoptosis is initiated by ligation of death receptors, through the extrinsic pathway, the upstream signals often require amplification by the mitochondrial pathway. Thus, MOMP is frequently the decisive event preceding cell death.

$\mathrm{BCL}-2$ family proteins are distinguished by four conserved $\mathrm{BCL}-2$ homology $(\mathrm{BH} 1-\mathrm{BH} 4)$ domains. In particular, the $\mathrm{BH} 3$ domain is invariably present and has an essential role in regulating cell death. BCL-2 family proteins engage in a complex network of heterodimeric interactions that collectively make life and death decisions for the cell. Anti-apoptotic family members (e.g. BCL-2 and BCL-XL) protect the cell against apoptotic stimuli, whereas pro-death proteins (BAX and BAK - one or the other is needed for apoptosis) actively kill the cell by inducing MOMP. Their functions are regulated by a third class of BCL-2 family members, the BH3-only proteins.

One such BH3-only protein, BID, provides a key link between the extrinsic and mitochondrial pathways to cell death because it is activated when extracellular stress cues, transmitted through cell surface death receptors, activate the protease Caspase-8, which cleaves BID into an inactive $\mathrm{N}$-terminal fragment and a critical pro-death C-terminal fragment (tBID). tBID and the other BH3-only proteins BIM and PUMA have two main functions: first, they can form stable heterodimers with anti-apoptotic family members such as $\mathrm{BCL}-\mathrm{XL}$, neutralizing their protective activity, and second, they can transiently interact with $\mathrm{BAX}$ and BAK, directly activating them. ${ }^{10,25-39}$ Other $\mathrm{BH} 3$-only proteins (e.g. BAD) can only suppress anti-apoptotic family members. The antiapoptotic family members act in two ways: to antagonize the multidomain proteins $\mathrm{BAX}$ and $\mathrm{BAK}$ and to sequester the BH3-only proteins.

\section{What Regulates the Interplay of Soluble Cytoplasmic BCL-2 Family Proteins with Membranes?}

Structural and biochemical studies have identified some of the early events in BAX and BAK occurring upon interaction with $\mathrm{BH} 3$ domain peptides. ${ }^{35,36,38,39}$ However, interactions among full-length BCL-2 family proteins normally take place in the membrane environment. This is obviously true for BAK, which is permanently membrane-resident. Nevertheless, some experimental alterations of the BAK molecule increase its solubility or decrease its MOM-association, even causing it to be inducibly MOM-translocated, much like BAX. ${ }^{40}$ With regard to $\mathrm{BAX}$, experiments in protein-free, cardiolipin-containing liposomes showed that tBID becomes recruited to the membrane before $B A X,{ }^{41}$ suggesting that initially, MOMassociated tBID might promote BAX accumulation in the MOM by evoking a conformational change needed for membrane integration. The MOM protein Mtch2 has been proposed as a tBID-binding receptor in the MOM. ${ }^{42}$ However, a recent study $^{43}$ has shown that Mtch2 accelerates the separation of the $\mathrm{N}$ - and $\mathrm{C}$-terminal fragments of Caspase-8cleaved BID and promotes a complex process of tBID integration in the membrane. This would then trigger BAX recruitment to the MOM and activation.

However, activation by tBID and other $\mathrm{BH}$-only proteins appears not to be the only trigger for BAX translocation. Recent studies in epithelial cells ${ }^{44}$ confirmed other studies in fibroblasts ${ }^{45,46}$ showing that, under nonapoptotic conditions, $\mathrm{BAX}$ exists in equilibrium between cytoplasmic and MOMassociated forms. A recent study by Schellenberg et al. ${ }^{44}$ now shows that in epithelial cells, detachment from the extracellular matrix causes a loss of adhesion-dependent survival signaling; somehow, this reduces the rate of BAX membrane dissociation, thereby shifting the equilibrium in favor of $\mathrm{MOM}$ localization. However, if cells are allowed to reattach before they become committed to apoptosis, MOM-associated BAX can move back to the cytoplasm. This reversibility of MOM localization suggests that BAX can be stably associated with mitochondria even in a non-activated state. Together, the retrotranslocation studies show that at least one form of BAX 
recruitment to the MOM does not appear to depend on full apoptotic activation by $\mathrm{BH} 3$-only proteins.

How BAX reversibly associates with the MOM is still not understood. As the BAX S184V mutant displays a lower rate of dissociation from the MOM, Schellenberg et al. ${ }^{44}$ suggest that the C-terminal $\alpha$-helix of wt BAX can interact weakly with the membrane. In vitro, BAX does interact transiently with liposomes, causing it to display the $6 \mathrm{~A} 7$ epitope. $^{47}$ This membrane-induced BAX conformational change is not inhibited by BCL-XL. Mixing BAX alone with liposomes does not lead to $\mathrm{BAX}$ oligomerization or even to measurable $\mathrm{BAX}$ membrane association, as measured by a float-up assay. For stable BAX integration, full activation (e.g. by tBID) is required. In principle, membrane integration, which would involve at least the hydrophobic $\mathrm{C}$ terminus and potentially other regions of this protein, is likely irreversible, as the energy barrier for removal of a transmembrane helix from the membrane to water would be very high. Within cells, the MOM-association equilibrium could instead involve a reversible interaction of BAX with resident MOM proteins, especially as adhesiondependent signaling alters this equilibrium.

We can well imagine that BAX (retro-)translocation and activation in whole cells involves multiple steps and stillunknown mechanisms. Anoikis in mammary epithelial cells involves an hours-long interval between BAX translocation to mitochondria and $B A X$ activation. In this setting, instead of the previously reported ability of $B C L-X L$ and $B A X$ to retrotranslocate each other, ${ }^{45} \mathrm{BCL}-\mathrm{XL}$ stabilizes $\mathrm{BAX}$ association with the MOM. Perhaps this reflects co-integration of these two proteins, as in the 'embedded together' scenario previously described by Andrews et al. ${ }^{14}$ Similarly, Llambi et al. $^{48}$ found that when BAX-induced MOMP is blocked by $\mathrm{BCL}-\mathrm{XL}$, both proteins become membrane-integrated. We do not understand what determines co-integration versus coretrotranslocation. In particular, the structural details of how these proteins interact in the membrane environment are yet unknown. However, one recent study dealt with the contribution of the C-terminal helix of BCL-XL, which is necessary for the ability of $B C L-X L$ to retrotranslocate $B A X .{ }^{46}$ Interestingly, replacing the $\mathrm{C}$ terminus of $\mathrm{BCL}-\mathrm{XL}$ with the corresponding portion of BAX retained the property of the chimeric molecule to interact reversibly with the MOM, but eliminated the ability to retrotranslocate BAX.

\section{Molecular Basis for BCL-2 Family Protein Function}

The molecular structures of BCL-XL, ${ }^{49} \mathrm{BAX}^{50}$ and BID, ${ }^{51,52}$ all determined in solution by NMR, provided the initial framework for understanding the functions and interactions of the BCL-2 family proteins. Despite their opposing functions, all three proteins adopt a common fold, with two central, more hydrophobic $\alpha$-helices forming a hairpin surrounded by six to eight more polar $\alpha$-helices (Figure 1). This structure appears to be shared by all other pro- and anti-apoptotic BCL-2 family members. ${ }^{53,54}$ The prototypical BCL-2 fold also resembles that of the pore-forming domains of bacterial toxins, suggestive of a common pore-forming function in which the two central helices $(\alpha 5-\alpha 6$ in BAX) are likely important for membrane insertion. ${ }^{55-57}$

The $\mathrm{BH} 1, \mathrm{BH} 2$ and $\mathrm{BH} 3$ domains form a hydrophobic groove on the protein surface that is conserved across both anti- and pro-apoptotic BCL-2 family members and is crucial for mediating their interactions. In $\mathrm{BCL}-\mathrm{XL}$, this canonical groove (binding cleft) serves as the binding site for the $\mathrm{BH} 3$ domains of its pro-apoptotic BCL-2 partners, including BAX/ BAK and BID. ${ }^{58}$ This interaction produces a stable complex that is thought to protect against cell death by effectively sequestering the death agonists. In the cytosolic form of BAX, however, the groove is normally occupied by BAX's own hydrophobic C-terminal helix $(\alpha 9)$, and certain BH3-only proteins (BID, BIM and perhaps PUMA) appear to interact with a different BAX site, diametrically opposed to the canonical BH3-binding groove. ${ }^{35}$ This interaction is weak, but it is needed to trigger BAX activation. BAK lacks this alternative site and appears to be activated by binding the $\mathrm{BH} 3$ domain of either BID or BIM to its canonical groove. $^{38,39,59}$

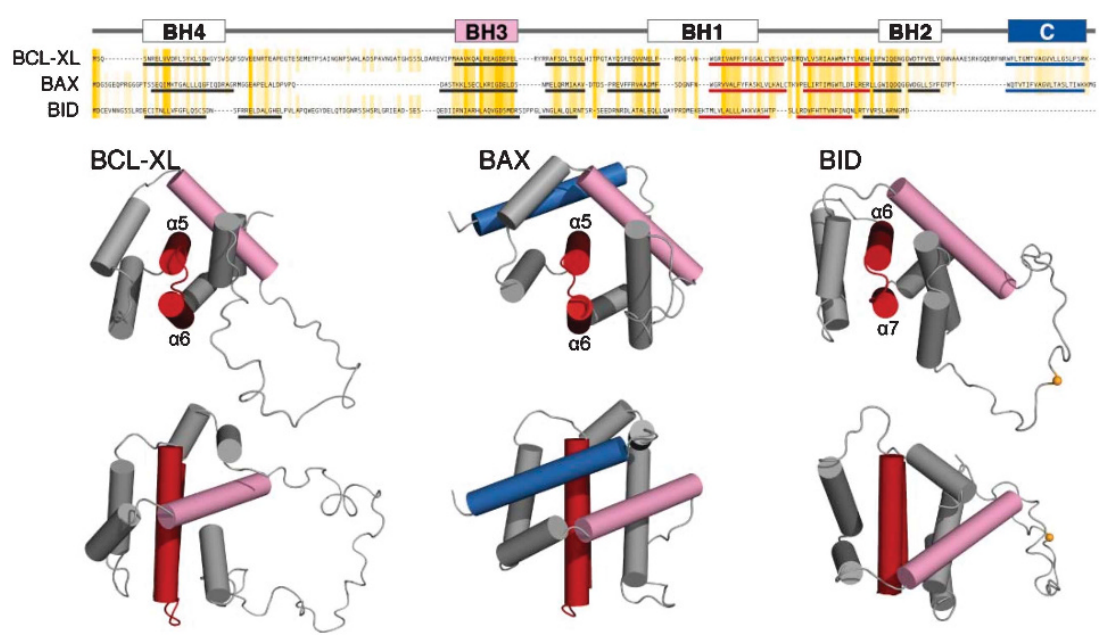

Figure $1 \mathrm{BCL}-2$ homology and solution NMR structures of $\mathrm{BCL}-\mathrm{XL},{ }^{49} \mathrm{BA} \mathrm{X}^{50}$ and $\mathrm{BID}$. $^{51,52}$ The proteins contain up to four $\mathrm{BH}$ domains and a 20 -residue hydrophobic $\mathrm{C}$ terminus. The $\mathrm{C}$ terminus of $\mathrm{BCL}-\mathrm{XL}$ was truncated to promote solubility. The BID caspase-8 cleavage site (Asp60) is denoted by a gold sphere. BH3 domains are shown in pink, hydrophobic $\mathrm{C}$ termini in blue and central membrane-inserting helices in red 
Recent structural studies show that truncated $B A X \Delta C$ and $\mathrm{BAK} \triangle \mathrm{C}$ (lacking the hydrophobic $\mathrm{C}$-terminal helix) can bind activator $\mathrm{BH} 3$ peptides through their respective $\mathrm{BH}$-binding clefts. ${ }^{39,60}$ BAX $\triangle \mathrm{C}$ treated with the detergent CHAPS and $\mathrm{BID}, \mathrm{BIM}$ or BAX BH3 peptides forms a domain-swapped dimer $^{60}$ similar to that formed by BCL-XL $\Delta C$ exposed to high $\mathrm{pH}$ or heat. ${ }^{61,62}$ Dimers induced by the detergent octylmaltoside yielded indistinguishable structures without the bound peptide. In these domain-swapped dimers, the $\alpha 5-\alpha 6$ helices of each monomer form one long extended helix and interact with the $\alpha 6-\alpha 5$ helices of the second monomer, forming a planar surface that is proposed to engage the membrane. A mutant form of BAK, rendered soluble by mutagenesis of its $\mathrm{C}$ terminus, also binds $\mathrm{BH} 3$ peptides through its surface cleft, $^{38}$ as does a highly truncated form of BAX spanning only helices $\alpha 2$ to $\alpha 5{ }^{60} \mathrm{BH} 3$ ligand association with the BAX or BAK surface cleft is distinct from its interaction with a diametrically opposed $\mathrm{BH} 3$ interaction site observed by Gavathiotis et al. ${ }^{35}$ for intact, monomeric BAX in solution, suggesting that $\mathrm{BAX}$ and $\mathrm{BAK}$ may each be activated by direct interaction with a $\mathrm{BH} 3$ ligand at distinct molecular sites, and that activation likely involves multiple steps. ${ }^{63}$

All of these studies suggest very interesting hypotheses about BCL-2 protein function. However, because they were performed with extensively truncated proteins, in the presence of detergents and far removed from the natural environment of the phospholipid bilayer membrane - conditions that can produce distortions in many integral membrane and membrane-associated proteins ${ }^{64}$ - it is unclear where and how these structures fit into the apoptosis pathways.

Cell-based and biophysical studies focused on BAK and $\mathrm{BAX}$ activation indicate that the $\mathrm{BH}$-groove interaction is essential for $\mathrm{BAK}^{65}$ and $\mathrm{BAX}^{66}$ oligomerization. However, the structures of the domain-swapped dimers appear at odds with data on the interfaces in BAX oligomers formed during apoptosis ${ }^{66-68}$ and incompatible with a model in which helices $\alpha 5$ and $\alpha 6$ insert into the membrane during permeabilization. The similarity of the $\mathrm{BAX} \Delta \mathrm{C}$ structures with pro-survival proteins, together with the facts that BAX inserts in membranes can form membrane pores as a monomer and is likely to unfold on the membrane (see below), indicate that swapped-dimer structures may not be directly involved in the activation pathway. However, they may represent some off-pathway conformations, possibly related to the reversible retrotranslocation process. The latter would be an attractive possibility because the blockage of helices $\alpha 5$ and $\alpha 6$ through the domain-swapping mechanism would prevent insertion of these helices into the membrane and thus prevent the subsequent, irreversible MOMP. Structural studies on fulllength proteins in the presence of a fully functional membrane bilayer environment will be needed to obtain a full picture of BAX/BAK activation.

\section{How does the BCL-2 Family Regulate MOMP?}

For MOMP to occur, BAX and BAK require direct activation by a 'direct activator' BH3-only protein, for example, BID, BIM or PUMA. ${ }^{34-36,38,39,59,69,70}$ Anti-apoptotic BCL-2 family proteins can block MOMP both by sequestering $\mathrm{BH}$-only proteins and by neutralizing BAX/BAK. This inhibitory cytoprotective function can in turn be neutralized by an excess of 'indirect activator' BH3-only proteins (e.g. BAD), which bind the antiapoptotic BCL-2 proteins, thereby liberating the pro-apoptotic direct activator BH3-only proteins. Some chemotherapeutic drugs currently under development (e.g. ABT-263, Navitoclax) act in a similar manner to antagonize the interactions of $\mathrm{BCL}-\mathrm{XL}$ and other anti-apoptotic relatives with their proapoptotic partners, thereby promoting apoptosis. ${ }^{71,72}$

After BAX activation via a transient hit-and-run interaction with a direct activator $\mathrm{BH} 3$-only protein such as tBID, BAX typically translocates from its soluble cytoplasmic state to a MOM-integrated state. BAK is constitutively membraneresident, but also becomes activated by BH3-only proteins in a similar manner. Activation of BAX and BAK leads to complete membrane insertion of these proteins and then to MOMP, which generally commits the cell to death. The early stages of BAX and BAK activation have been analyzed elegantly by structure-guided mutational analysis. ${ }^{35,36,38,39}$ However, as the structures of membrane-inserted BAX and BAK are not known, the exact conformational changes in these proteins associated with MOMP activity are still unclear.

Indeed, the limited structural information that is available for the membrane-associated states of BCL-XL, BAX and tBID ${ }^{50,73-78}$ provide only a glimpse of how BCL-2 proteins interact with membranes. tBID has been shown to adopt a stable helical conformation in lipids, with its helices parallel to the membrane surface and no transmembrane helix insertion. $^{75,79}$ BAX-derived peptides corresponding to $\alpha 5-\alpha 6$ readily insert into membranes and mediate the formation of protein-lipidic pores. ${ }^{80-83}$ The conformation of BCL-XL in lipid bilayers may involve membrane embedding of the $B C L-X L$ helical hairpin $(\alpha 5-\alpha 6)$ as well as helix $\alpha 1{ }^{73,74}$ However, these results were obtained using truncated $B C L-X L$ lacking the hydrophobic $\mathrm{C}$ terminus (BCL-XL $\Delta \mathrm{C}$ ), or using detergents to either solubilize or activate a conformational change, manipulations that can significantly distort protein structure and function. ${ }^{64}$ Indeed, a number of recent reports have demonstrated the importance of studying membrane-associated proteins, including BCL-2 family proteins, in a lipid membrane environment. ${ }^{84,85}$

\section{BAX Monomers are a Driving Force for Membrane Perturbation}

Major conformational rearrangements and extensive homooligomerization of BAX and BAK are widely assumed to be necessary for pore formation and MOMP. ${ }^{50}$ However, a detailed spatiotemporal molecular mechanism for these events remains elusive. Until recently, it has not been possible experimentally to separate BAX/BAK oligomerization from the pore-formation activity involved in MOMP, and so researchers have assumed that oligomerization is a requirement. However, two independent investigations have now converged on the notion that integrated BAX monomers are sufficient to perturb the lipid bilayer.

In recent electron cryomicroscopy (cryo-EM) studies, Volkmann and co-workers ${ }^{78}$ dissected the structural correlates of BAX membrane insertion, in a situation where oligomerization is physically impossible. This was accomplished through the use of nanometer-scale phospholipid 
bilayer islands (nanodiscs), each capable of accommodating only a single BAX molecule. ${ }^{78}$ The three-dimensional reconstructions of these membrane assemblies (Figure 2) demonstrate that in the presence of a BID BH3 peptide, an individual BAX molecule can insert into the nanodisc membrane, perturb the lipid bilayer and form a pore of $\sim 3.5 \mathrm{~nm}$ in diameter. While the precise structure of this BAX pore will have to be determined, its monomeric state is dictated by the small, discrete size of the lipid bilayer nanodiscs, which can only accommodate a single membrane-inserted BAX. Native gels, fluorescence and immunoblotting data all confirmed that BAX was monomeric in the nanodisc under the conditions used for imaging.

Interestingly, the three-dimensional structure of this fulllength BAX-membrane assembly contained no discernible, compact, extra globular density that would account for a folded BAX monomer globular structure associated with the nanodiscs (Figure 2). This is in stark contrast to other recent nanodisc studies where integrated proteins such as integrin receptors, anthrax toxin or SNARE proteins are clearly visible. ${ }^{86-88}$ This observation suggests that BAX is unfolded on top of the nanodisc surface (Figure 3 ), presumably with its amphiphilic portions inserted into the nanodiscs in a similar way to the 'umbrella model' proposed for colicin, where the outer helices of the globular structure rearrange to form the 'umbrella' resting on the membrane surface, while the central hydrophobic helical hairpin (corresponding to $\alpha 5-\alpha 6$ in BAX) inserts across the membrane to form the 'umbrella handle'. ${ }^{89-91}$ Such unfolding is consistent with the finding that a substantial structural reorganization of BAX occurs during BH3-triggered activation. ${ }^{36}$ a
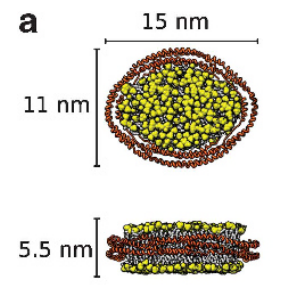

Model
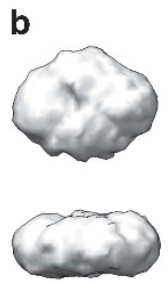

Nanodisc (ND)
C
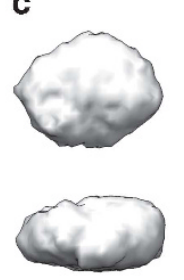

$\mathrm{ND}+\mathrm{BH} 3$

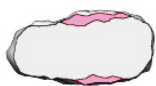

d
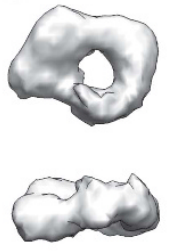

$\mathrm{ND}+\mathrm{Bax}+\mathrm{BH} 3$

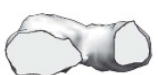

Figure 2 Three-dimensional cryo-EM reconstructions of BAX nanodisc assemblies. $^{78}$ (a) Model of nanodisc derived from the three-dimensional density. The stabilizing peptide belt is shown as cartoon representation in orange. The lipid bilayer is shown in yellow (polar headgroups) and gray (hydrocarbon tails). (b-d) 3D reconstructions of the nanodiscs (ND) are shown as gray surface representations. The contour level was chosen to accommodate the length of the protein belt in the circumference of the density. Two orthogonal views are shown; the bottom row shows the density cut open at the center (b) Empty nanodiscs. (c). Nanodisc in the presence of BID BH3 peptides. Note the additional thickness if compared with (b), attributable to the density of BID BH3 peptides (unknown stoichiometry) bound to the nanodisc. The increase in thickness is mapped onto the cut-open view (bottom row) in pink. The binding of BID BH3 to nanodiscs was independently verified with gel electrophoresis and fluorescence tagging. (d) Nanodisc in the presence of BAX and BID BH3 peptides showing significant distortions of the bilayer and a pronounced hole in the nanodisc. Binding of single BAX monomers was independently verified using gel electrophoresis and fluorescence tagging
In contrast to these small BAX monomer-induced pores, Bax-dependent permeabilization of the MOM involves a much greater permeability of the membrane, allowing the efflux of macromolecules such as Smac/DIABLO dimers or very large dextran molecules. ${ }^{10}$ How this permeabilization occurs is a key question. Judging from the sizes of the released macromolecules, up to $2000 \mathrm{kD}$, we can infer that MOMP involves a large-scale rearrangement of the membrane. Paradoxically, however, traditional EM methods did not reveal any alterations in the MOM. ${ }^{10}$ This suggested the possibility that MOMP arises not from the formation of fixed proteinaceous pores but rather from pores that are lipidic (and not easily preserved during embedding for EM). Indeed, when Baxinduced pores in small unilamellar vesicles ${ }^{92}$ and in bacteria ${ }^{93}$ were later imaged using gentler cryo-EM techniques, they were seen to be large and heterogeneous (25-100 nm), much like those produced by bacterial toxins. Similar large Baxinduced pores have now been imaged in purified MOMs by cryo-EM (Newmeyer and Kuwana, personal communication). These and other observations ${ }^{80,82,94}$ argue that MOMP is the result of lipidic pore formation.

Indeed, the pores produced in nanodiscs by single Bax molecules are already lipidic in nature. The number of helices that can conceivably insert into the membrane from a single BAX molecule (four) is too small to allow complete protein lining of a 3.5-nm diameter pore ${ }^{78}$ (Figure 3). The formation of lipidic pores induced by BAX monomers in the initial stages of MOMP is an attractive alternative to models where the initial pores are protein-lined and formed by ordered BAX oligomers. The minimal protein requirement of monomer-induced pores potentially favors faster induction of the pro-death signaling cascade, similar to the efficient process implemented by bacterial toxins. The joining of the initial monomer-induced lipidic pores at later stages of MOMP to increase pore size can be achieved with far less conformational adaption than the joining of protein-lined pores would require.

Independent evidence from biochemical kinetic analysis now implies that Bax monomers are important for the formation even of large protein-conducting pores. Newmeyer and co-workers ${ }^{95}$ showed that MOMP kinetics are linearly dependent on BAX concentration and show neither cooperativity nor saturability relative to BAX (Figures 4 and 5). This implies that BAX monomers, not oligomers, drive the kinetics of pore formation. Together, the results of the nanodisc cryo-EM and kinetic experiments argue that monomeric membrane-inserted BAX is the key functional unit responsible for initiating MOM pore formation, MOM destabilization and MOMP, leading to apoptosis.

How do the small membrane perturbations produced by individual Bax monomers lead to the formation of supramolecular pores? The mechanism is still unclear. One possibility is that an abundance of integrated BAX monomers in a local membrane region could produce enough curvature stress to destabilize the membrane. Several reports have argued that curvature stress is the ultimate mechanism promoting the formation of protein-conducting membrane pores, and quite possibly the process can involve a cooperation between protein and lipid components to sculpt the membrane. ${ }^{94,96-100}$ Indeed, in cryo-EM images, Bax-induced pores in liposomes ${ }^{92}$ and MOM vesicles (Kuwana and Newmeyer, personal 
a

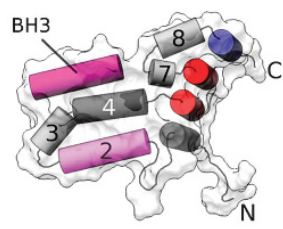

b
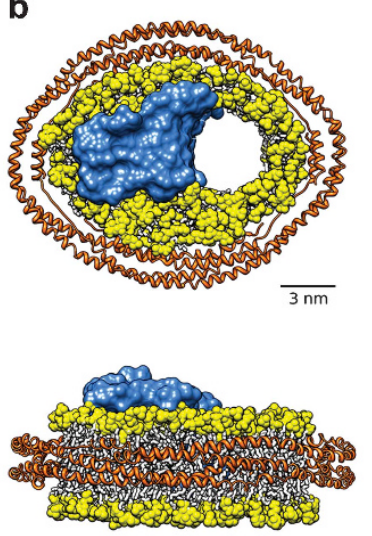

C
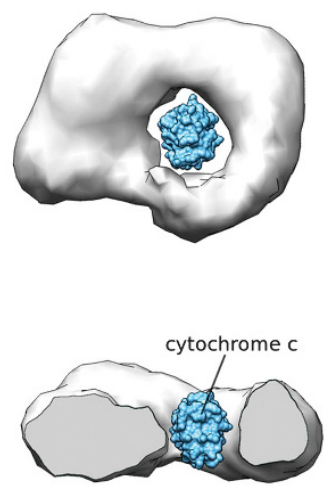

d
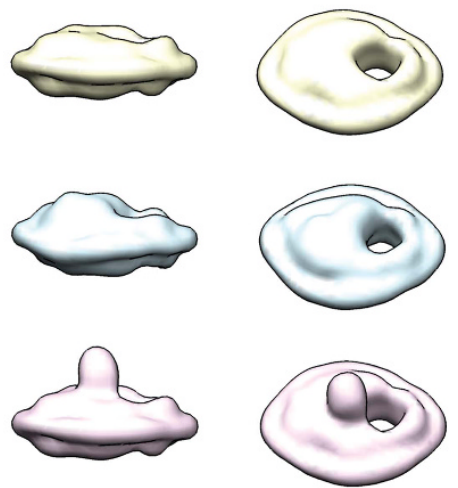

Figure 3 BAX monomers unfold when inserted into the membrane bilayer. (a) Tentative model of membrane-inserted BAX with BID BH3 peptide derived from the cryo-EM data. $^{78}$ The $\mathrm{N}$ - and $\mathrm{C}$ termini are marked. The helices are represented as cylinders; the molecular surface is shown as a transparent surface. The numbers correspond to the numbering of the helices following the sequence. Helices $\alpha 1, \alpha 5, \alpha 6$ and $\alpha 9$ are modeled to insert into the membrane and to line the pore. This configuration would be the "bestcase scenario' for pore lining and minimizing the material that needs to be placed on the surface. The top view is perpendicular to the membrane plane and the bottom view is parallel to the membrane. (b) BAX/BID peptide (blue surface) modeled onto the nanodisc (yellow) containing a 3.5-nm hole. There is only enough space for a single BAX molecule and there are not enough helices inserted for lining the entire pore. (c) Cryo-EM reconstruction of nanodisc in the presence of BAX and BID peptide (white surface) with the structure of cytochrome $c$ (blue surface) modeled into the hole. The hole is big enough to allow cytochrome $c$ to pass. (d) Densities calculated at 2.5 -nm resolution from a model of nanodisc with a 3.5-nm hole (yellow, top row), the model shown in (b) (blue surface, center row) and a model of a nanodisc with 3.5-nm hole with the globular solution structure of BAX attached to the surface. The view in the first column is parallel to the membrane plane and the view in the second column is tilted by $45^{\circ}$ downwards around the horizontal axis
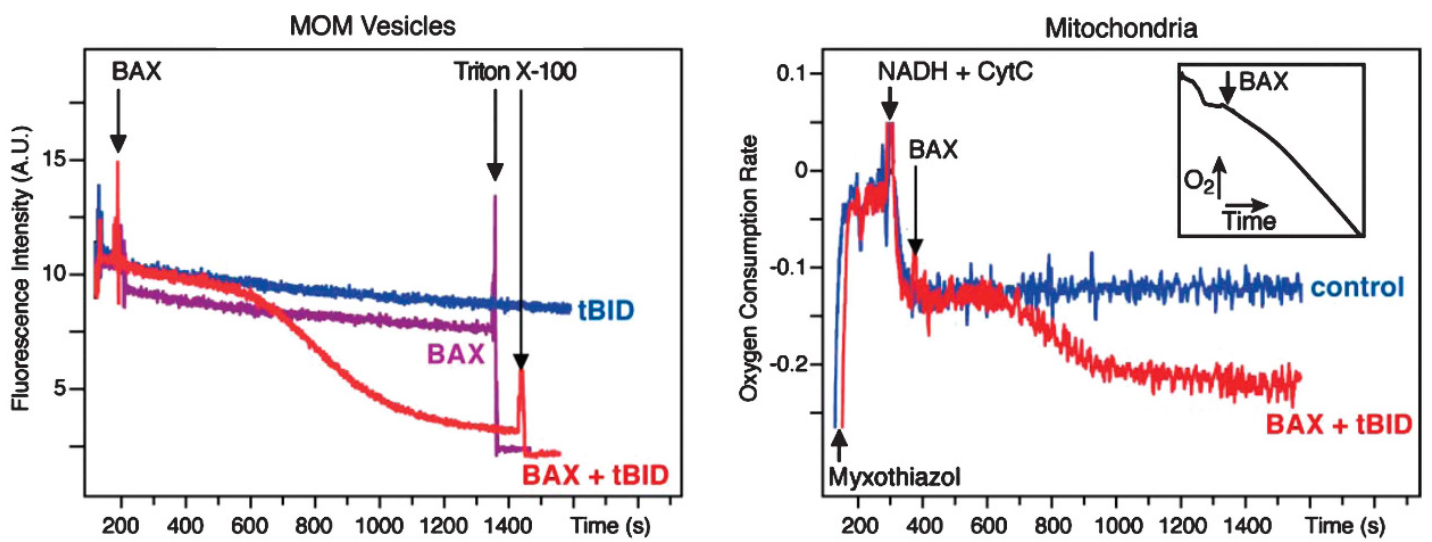

Figure 4 MOM vesicles and mitochondria display similar biphasic permeabilization kinetics upon treatment with recombinant BAX activated by cleaved BID (tBID). ${ }^{95}$ (a) Purified MOM vesicles were pre-loaded with fluorescein-dextrans and dextran content was measured by fluorescence intensity in the presence of anti-fluorescein quenching antibody. (b) Permeabilization of whole mitochondria was measured as the rate of oxygen consumption driven by exogenous cytochrome $c$. Inset shows total oxygen; main curves are the derivatives of oxygen traces with respect to time. Note that in both cases, there is an initial lag phase (absent in liposomes; not shown) followed by an exponential decay phase

communication) are frequently adjoined by negatively curved membrane regions. This strongly suggests a pore formation or enlargement mechanism involving curvature stress. An alternative model was proposed by Martinez et al. ${ }^{101}$ after observing a BAX-dependent stepwise opening of highconductance membrane channels. Possibly, integrated BAX monomers could cluster together in a quantum fashion to form ever-larger pores ringed by BAX molecules. These models need not be mutually exclusive. Cryo-EM imaging of extended lipid bilayer membranes sufficiently large to accommodate several BAX molecules, together with additional structure determination approaches, will ultimately be needed to determine the membraneassociated structures of membrane-embedded BAX oligomers at a high resolution. Imaging of colloidal goldlabeled BAX molecules in the MOM, if feasible, might also provide information about the organization of BAX molecules around the lipidic pores.

How BAX oligomerization might have a role in this process is still unclear. Based on the universal finding so far that BAX/ BAK mutants blocking oligomerization also block MOMP, it is tempting to conclude that oligomerization is required for pore formation. One common model is that the pores are enlarged through a process of Bax autoactivation, in which BAX oligomers at the pore rim catalyze the integration of free BAX monomers and their addition to the ends of existing oligomers. However, to explain the linear response of MOMP kinetics to BAX concentrations, the effect of oligomerization, if 

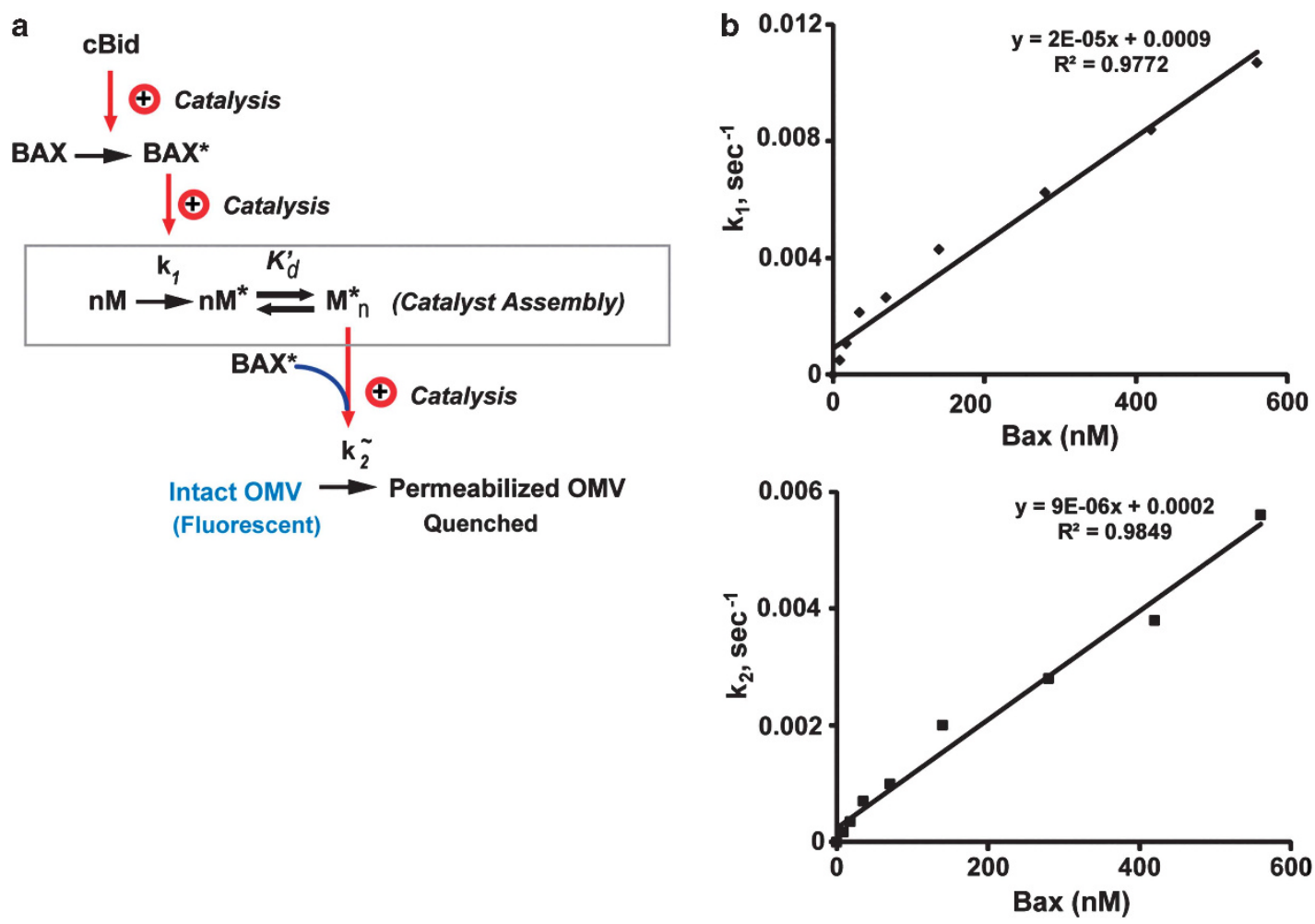

Figure 5 Kinetic analysis shows that BAX-induced permeabilization of MOM vesicles involves the assembly of a non-BAX oligomeric catalyst that facilitates BAX-induced pore formation. ${ }^{95}$ (a) MOMP reaction diagram inferred from kinetic analysis. Measured MOMP kinetics as shown in Figure 5 are most simply explained by the existence of two coupled reaction tiers: (i) BAX-induced formation of an oligomeric catalyst $\mathrm{M}_{n}^{*}$, with rate $\mathrm{k}_{1}$ and $n \geq 12$, which occurs during the first kinetic phase (lag), shown in the gray box and (ii) BAX-induced pore formation, with rate $k_{2}$, which occurs during the second kinetic phase (permeabilization). (b) The measured kinetic constants $k_{1}$ and $k_{2}$ depend linearly on BAX concentration, implying that BAX acts noncooperatively and thus that the oligomeric catalyst $\mathrm{M}$ is distinct from $\mathrm{BAX}$

any, would have to be non-rate-limiting, that is, very rapid. ${ }^{95}$ This is formally possible. Nevertheless, a requirement for oligomerization has not been proven. Although oligomerization is an inevitable byproduct of $\alpha$-helix insertion, it might not contribute to membrane destabilization, but perhaps only to the autoactivation of free Bax monomers. Oligomers could have a function different from pore formation, for example, to regulate mitochondrial dynamics. Alternatively, oligomers could be inert or even inhibitory to pore formation. In this way, oligomers could act as a brake to limit the runaway amplification of a sub-threshold apoptotic signal. If so, cell death would require the sustained production of integrated BAX/BAK monomers to promote pore formation and enlargement.

As mentioned, BAX by itself can form pores in liposome membranes, upon activation by a $\mathrm{BH} 3$-domain peptide or a BH3-only protein. However, to what extent this intrinsic activity explains MOM permeabilization in apoptotic cells remains unclear. Highlighting this uncertainty, the recent study by Kushnareva et al..$^{95}$ showed that native MOMs are more responsive to low concentrations of BAX than liposomes and display more complex, biphasic permeabilization kinetics (Figure 5). This enhanced BAX response requires the participation of heat-labile MOM proteins. Furthermore, these authors found that, in order to fit the measured kinetic curves, it was necessary to consider a twostage reaction scheme. In the first stage, activated BAX triggers the assembly of a non-Bax oligomeric complex. In the second stage, this complex catalyzes the BAXdependent formation of membrane pores. Interestingly, the catalyst formation reaction exhibited a membrane phase-like transition near $28^{\circ} \mathrm{C}$, suggesting that it involves a localized membrane-remodeling event.

The behavior of this catalyst complex is reminiscent of the membrane-distorting activities of Drp1 and Bif-1, two proteins previously proposed to have roles in MOMP. ${ }^{99,102-104}$ However, Kushnareva et al. ${ }^{95}$ found no evidence supporting a role for these proteins in Bax-induced permeabilization of isolated MOM vesicles. Chemical compounds (mDivi-1 analogs) that had been shown to inhibit Drp1's yeast ortholog, Dnm1, also inhibit MOMP in mammalian cells, ${ }^{105}$ and some mDivi-1 analogs blocked BAX-induced pore formation in rat liver and Xenopus MOM vesicles. Kushnareva et al. ${ }^{95}$ were unable to detect Drp1 in their MOM preparations, suggesting that the catalyst protein whose existence was inferred by their studies is different from Drp1. Although Bif-1 was weakly detectable in rat liver MOMs, it was below the limits of immunoblot detection in Xenopus egg MOM vesicles, which display the same type of MOMP kinetics as rat liver MOM vesicles. Naturally, these results do not exclude the possibility that Drp1 or Bif-1 participate in MOMP within cells, as these proteins could be recruited from the cytoplasm. However, the cell-free studies argue that Drp1 and Bif-1 are not essential for MOMP and that another unidentified MOM-resident protein catalyzes BAX-induced MOMP. In general, the auxiliary roles of non-BCL-2 family proteins in MOMP remain to be clarified. 
Nevertheless, the simplest hypothesis is that such proteins facilitate BAX's intrinsic pore-forming function.

\section{How does BCL-XL Protect Against Cell Death?}

BCL-XL protects cells both by interacting with BAX to prevent its membrane integration and by interacting with BID and other BH3-only proteins, to sequester their pro-death activity. The $\mathrm{C}$ terminus of $\mathrm{BCL}-\mathrm{XL}$ contains a clear MOM-targeting motif and shares sequence homology with those of BAX, BAK and other MOM-targeted BCL-2 proteins. The lipid bilayer membrane strongly influences BCL-2 protein-protein interactions, ${ }^{20,48,84,106}$ and several lines of evidence show that the hydrophobic $\mathrm{C}$ terminus of $\mathrm{BCL}-\mathrm{XL}$ is important for regulating both the interaction of $B C L-X L$ with its partners and its membrane integration. However, most structural studies have focused on truncated forms of BCL-XL lacking the hydrophobic $C$ terminus, to promote solubility, and little is known about how BCL-XL interacts with the membrane or with membrane-associated BAX and BID.

Solid-state NMR studies suggest an 'umbrella' model of $\mathrm{BCL}-\mathrm{XL}$ membrane integration, ${ }^{74}$ as proposed for colicins. ${ }^{89-91}$ However, the studies with $\mathrm{BCL}-\mathrm{XL}$ were performed with truncated protein, and the presence of the $C$ terminus is likely to have a significant effect on the membraneassociated conformation of this protein. The structure of BAX is the only one determined for an intact, multidomain BCL-2 protein including the hydrophobic $\mathrm{C}$ terminus. ${ }^{50}$ In solution, this membrane-targeting module folds into BAX's BH3binding cleft, suggesting that it may act as a regulatory switch for intermolecular interactions. Recent structural studies with moderately truncated forms of anti-apoptotic BCL-W indicate that it also may adopt a similar conformation. ${ }^{107,108}$ Thus, the $\mathrm{C}$ terminus of $\mathrm{BCL}-\mathrm{XL}$ is also likely to interact with the $\mathrm{BH} 3-$ binding cleft, with important consequences for the interactions of $B C L-X L$ with its partners and with membranes.

Recently, BCL-XL and BAX have been reported to cycle between cytosolic and MOM-associated states, and each protein can cause the other to retrotranslocate from the MOM back to the cytoplasm. ${ }^{45,46}$ The mechanism of retrotranslocation is still unknown. The simplest hypothesis is that the mutual membrane repulsion of $B C L-X L$ and $B A X$ results from the intrinsic interactions of these proteins in the membrane. Although the presence of an activating $\mathrm{BH}$-only protein would be required for the full integration of $B A X$ in the membrane, even the unactivated BAX molecule can interact weakly with the membrane, perhaps through its $\mathrm{C}$ terminus. ${ }^{47,109}$ If so, retrotranslocation may then result from competitive displacement of this weak membrane interaction of the Bax $\mathrm{C}$ terminus by interaction with the hydrophobic groove of a BCL-XL molecule (which would be exposed upon the transient interaction of this $B C L-X L$ molecule with the membrane). Afterwards, the resulting $B A X-B C L-X L$ heterodimer would dissociate from the membrane and then further dissociate into soluble monomers. Similarly, retrotranslocation of BCL-XL could involve a similar displacement of its C-terminal weak membrane interaction by the hydrophobic groove of Bax.

In the presence of a $\mathrm{BH} 3-$ only protein such as $\mathrm{tBID}$, the situation is different, as BAX and BCL-XL can become irreversibly membrane-integrated. In this situation, how $\mathrm{BCL}-\mathrm{XL}$ acts is still unclear, as in some cases $\mathrm{BCL}-\mathrm{XL}$ can block the membrane integration of $B A X$, while in other cases, $\mathrm{BCL}-\mathrm{XL}$ and $\mathrm{BAX}$ can both be membrane-integrated. For example, in the nanodisc cryo-EM study, Volkmann et al. ${ }^{78}$ found that $B C L-X L$ abolished the integration of $B A X$ into nanodisc membranes in the presence of BID BH3 peptides. This agrees with other work ${ }^{10,34,95,110-112}$ showing that BCL$\mathrm{XL}$ or $\mathrm{BCL}-2$ can block membrane recruitment and integration of $B A X$, even in the presence of a BAX-activating signal. In other studies, membrane integration of both $B C L-X L$ and $B A X$ (i.e. the 'embedded together' scenario ${ }^{14}$ ) was observed. ${ }^{44,48}$ Perhaps co-integration requires a balanced stoichiometry of $\mathrm{BCL}-\mathrm{XL}$ and $\mathrm{BAX}{ }^{113}$ Alternatively, it could require the participation of a MOM protein that facilitates insertion of $\mathrm{BAX}$ and $\mathrm{BCL}-\mathrm{XL}$, similar to the recently reported role of Mtch2 in facilitating the membrane integration of tBID. ${ }^{43}$

\section{Outlook}

Understanding the process of BAX/BAK-dependent membrane pore formation remains a Holy Grail of the apoptosis field. Recent publications significantly advance our understanding of the mitochondrion-dependent intrinsic pathway to apoptosis, but also emphasize the need for high-resolution structural studies aimed at determining the molecular conformations and interactions of key BCL-2 family proteins without truncations and within a membrane environment. This kind of detailed molecular understanding is needed to define the intrinsic activities of this protein family and also to facilitate analysis of the roles of cofactor proteins, once these are identified.

The evidence is overwhelming that the membrane environment is critical for preserving the structural and functional integrity of both integral and membrane-associated proteins. This is entirely consistent with Anfinsen's hypothesis that protein conformation 'is determined by the totality of interatomic interactions and hence by the amino acid sequence in a given environment'. ${ }^{109}$ For membrane-associated proteins such as the BCL-2 family proteins, the 'given environment' of the phospholipid bilayer is essential for preserving native structure and function.

\section{Conflict of Interest}

The authors declare no conflict of interest.

Acknowledgements. This work was supported by National Institutes of Health (NIH) grants P01-GM098412 (DH, NV), P01-Al074805 and R01-GM100265 (FMM) and R01-GM62289 (DDN).

1. Kerr JF, Wyllie AH, Currie AR. Apoptosis: a basic biological phenomenon with wide-ranging implications in tissue kinetics. Br J Cancer 1972; 26: 239-257.

2. Tsujimoto $Y$, Finger LR, Yunis J, Nowell PC, Croce CM. Cloning of the chromosome breakpoint of neoplastic B cells with the $t(14 ; 18)$ chromosome translocation. Science 1984; 226: 1097-1099.

3. Vaux DL, Cory S, Adams JM. Bcl-2 gene promotes haemopoietic cell survival and cooperates with c-Myc to immortalize pre-B cells. Nature 1988; 335: 440-442.

4. Boise LH, Gonzalez-Garcia M, Postema CE, Ding L, Lindsten T, Turka LA et al. bcl-x, a bcl-2-related gene that functions as a dominant regulator of apoptotic cell death. Cell 1993; 74: 597-608. 
5. Hockenbery DM, Oltvai ZN, Yin XM, Milliman CL, Korsmeyer SJ. Bcl-2 functions in an antioxidant pathway to prevent apoptosis. Cell 1993; 75: 241-251.

6. Newmeyer DD, Farschon DM, Reed JC. Cell-free apoptosis in Xenopus egg extracts: inhibition by $\mathrm{Bcl}-2$ and requirement for an organelle fraction enriched in mitochondria. Cell 1994; 79: 353-364.

7. Korsmeyer SJ. Regulators of cell death. Trends Genet 1995; 11: 101-105.

8. Kluck RM, Bossy-Wetzel E, Green DR, Newmeyer DD. The release of cytochrome c from mitochondria: a primary site for Bcl-2 regulation of apoptosis. Science 1997; 275: 1132-1136.

9. Yang J, Liu X, Bhalla K, Kim CN, Ibrado AM, Cai J et al. Prevention of apoptosis by Bcl-2: release of cytochrome c from mitochondria blocked. Science 1997; 275: 1129-1132.

10. Kuwana T, Mackey MR, Perkins G, Ellisman MH, Latterich M, Schneiter R et al. Bid, Bax, and lipids cooperate to form supramolecular openings in the outer mitochondrial membrane. Cell 2002; 111: 331-342.

11. Kuwana T, Newmeyer DD. Bcl-2-family proteins and the role of mitochondria in apoptosis. Curr Opin Cell Biol 2003; 15: 691-699.

12. Danial NN, Korsmeyer SJ. Cell death: critical control points. Cell 2004; 116: 205-219.

13. Adams JM, Cory S. Bcl-2-regulated apoptosis: mechanism and therapeutic potential. Curr Opin Immunol 2007; 19: 488-496.

14. Leber B, Lin J, Andrews DW. Embedded together: the life and death consequences of interaction of the Bcl-2 family with membranes. Apoptosis 2007; 12: 897-911.

15. Chipuk JE, Green DR. How do BCL-2 proteins induce mitochondrial outer membrane permeabilization? Trends Cell Biol 2008; 18: 157-164

16. Degterev A, Yuan J. Expansion and evolution of cell death programmes. Nature reviews 2008; 9: 378-390

17. Reed JC. Bcl-2-family proteins and hematologic malignancies: history and future prospects. Blood 2008; 111: 3322-3330.

18. Youle RJ, Strasser A. The BCL-2 protein family: opposing activities that mediate cell death. Nat Rev Mol Cell Biol 2008; 9: 47-59.

19. Yao Y, Marassi FM. BAX and BAK caught in the act. Mol Cell 2009; 36: 353-354.

20. Leber B, Lin J, Andrews DW. Still embedded together binding to membranes regulates Bcl-2 protein interactions. Oncogene 2010; 29: 5221-5230.

21. Martinou JC, Youle RJ. Mitochondria in apoptosis: Bcl-2 family members and mitochondrial dynamics. Dev Cell 2011; 21: 92-101.

22. Colell A, Ricci JE, Tait S, Milasta S, Maurer U, Bouchier-Hayes L et al. GAPDH and autophagy preserve survival after apoptotic cytochrome $c$ release in the absence of caspase activation. Cell 2007; 129: 983-997.

23. Lartigue L, Kushnareva Y, Seong Y, Lin H, Faustin B, Newmeyer DD. Caspaseindependent mitochondrial cell death results from loss of respiration, not cytotoxic protein release. Mol Biol Cell 2009; 20: 4871-4884.

24. Tait SW, Parsons MJ, Llambi F, Bouchier-Hayes L, Connell S, Munoz-Pinedo C et al. Resistance to caspase-independent cell death requires persistence of intact mitochondria. Dev Cell 2010; 18: 802-813.

25. Wang K, Yin XM, Chao DT, Milliman CL, Korsmeyer SJ. BID: a novel BH3 domain-only death agonist. Genes Dev 1996; 10: 2859-2869.

26. Gross A, Yin XM, Wang K, Wei MC, Jockel J, Milliman C et al. Caspase cleaved $\mathrm{BID}$ targets mitochondria and is required for cytochrome $\mathrm{c}$ release, while $\mathrm{BCL}-\mathrm{XL}$ prevents this release but not tumor necrosis factor-R1/Fas death. J Biol Chem 1999; 274: $1156-1163$.

27. Li H, Zhu H, Xu CJ, Yuan J. Cleavage of BID by caspase 8 mediates the mitochondrial damage in the Fas pathway of apoptosis. Cell 1998; 94: 491-501.

28. Luo X, Budihardjo I, Zou H, Slaughter C, Wang X. Bid a Bcl2 interacting protein, mediates cytochrome $c$ release from mitochondria in response to activation of cell surface death receptors. Cell 1998; 94: 481-490.

29. Eskes R, Desagher S, Antonsson B, Martinou JC. Bid induces the oligomerization and insertion of Bax into the outer mitochondrial membrane. Mol Cell Biol 2000; 20: 929-935.

30. Korsmeyer SJ, Wei MC, Saito M, Weiler S, Oh KJ, Schlesinger PH. Pro-apoptotic cascade activates BID, which oligomerizes BAK or BAX into pores that result in the release of cytochrome c. Cell Death Differ 2000; 7: 1166-1173.

31. Cheng EH, Wei MC, Weiler S, Flavell RA, Mak TW, Lindsten T et al. BCL-2, BCL-X(L) sequester $\mathrm{BH} 3$ domain-only molecules preventing BAX- and BAK-mediated mitochondrial apoptosis. Mol Cell 2001; 8: 705-711.

32. Scorrano L, Ashiya M, Buttle K, Weiler S, Oakes SA, Mannella CA et al. A distinct pathway remodels mitochondrial cristae and mobilizes cytochrome $\mathrm{c}$ during apoptosis. Dev Cell 2002; 2: 55-67.

33. Chen L, Willis SN, Wei A, Smith BJ, Fletcher Jl, Hinds MG et al. Differential targeting of prosurvival $\mathrm{Bcl}-2$ proteins by their $\mathrm{BH} 3-$ only ligands allows complementary apoptotic function. Mol Cell 2005; 17: 393-403

34. Kuwana T, Bouchier-Hayes L, Chipuk JE, Bonzon C, Sullivan BA, Green DR et al. BH3 domains of $\mathrm{BH} 3$-only proteins differentially regulate Bax-mediated mitochondrial membrane permeabilization both directly and indirectly. Mol Cell 2005; 17: 525-535.

35. Gavathiotis E, Suzuki M, Davis ML, Pitter K, Bird GH, Katz SG et al. BAX activation is initiated at a novel interaction site. Nature 2008; 455: 1076-1081.

36. Gavathiotis E, Reyna DE, Davis ML, Bird GH, Walensky LD. BH3-triggered structural reorganization drives the activation of proapoptotic BAX. Mol Cell 2010; 40: 481-492.
37. Du H, Wolf J, Schafer B, Moldoveanu T, Chipuk JE, Kuwana T. BH3 domains other than Bim and Bid can directly activate Bax/Bak. J Biol Chem 2011; 286: 491-501.

38. Leshchiner ES, Braun CR, Bird GH, Walensky LD. Direct activation of full-length proapoptotic BAK. Proc Natl Acad Sci USA 2013; 110: E986-E995.

39. Moldoveanu T, Grace CR, Llambi F, Nourse A, Fitzgerald P, Gehring K et al. BID-induced structural changes in BAK promote apoptosis. Nat Struct Mol Biol 2013; 20: 589-597.

40. Ferrer PE, Frederick P, Gulbis JM, Dewson G, Kluck RM. Translocation of a Bak $\mathrm{C}$-terminus mutant from cytosol to mitochondria to mediate cytochrome $\mathrm{C}$ release: implications for Bak and Bax apoptotic function. PLoS One 2012; 7: e31510.

41. Lovell JF, Billen LP, Bindner S, Shamas-Din A, Fradin C, Leber B et al. Membrane binding by tBid initiates an ordered series of events culminating in membrane permeabilization by Bax. Cell 2008; 135: 1074-1084.

42. Zaltsman Y, Shachnai L, Yivgi-Ohana N, Schwarz M, Maryanovich M, Houtkooper RH et al MTCH2/MIMP is a major facilitator of tBID recruitment to mitochondria. Nat Cell Biol 2010; 12: $553-562$.

43. Shamas-Din A, Bindner S, Zhu W, Zaltsman Y, Campbell C, Gross A et al. tBid undergoes multiple conformational changes at the membrane required for Bax activation. J Biol Chem 2013; 288: 22111-22127.

44. Schellenberg B, Wang P, Keeble JA, Rodriguez-Enriquez R, Walker S, Owens TW et al. Bax exists in a dynamic equilibrium between the cytosol and mitochondria to control apoptotic priming. Mol Cell 2013; 49: 959-971.

45. Edlich F, Banerjee S, Suzuki M, Cleland MM, Arnoult D, Wang C et al. Bcl-x(L) retrotranslocates Bax from the mitochondria into the cytosol. Cell 2011; 145: 104-116.

46. Todt F, Cakir Z, Reichenbach F, Youle RJ, Edlich F. The C-terminal helix of Bcl-x $(\mathrm{L})$ mediates Bax retrotranslocation from the mitochondria. Cell Death Differ 2013; 20: 333-342.

47. Yethon JA, Epand RF, Leber B, Epand RM, Andrews DW. Interaction with a membrane surface triggers a reversible conformational change in Bax normally associated with induction of apoptosis. J Biol Chem 2003; 278: 48935-48941.

48. Llambi F, Moldoveanu T, Tait SW, Bouchier-Hayes L, Temirov J, McCormick LL et al. A unified model of mammalian BCL-2 protein family interactions at the mitochondria. Mo Cell 2011; 44: 517-531.

49. Muchmore SW, Sattler M, Liang H, Meadows RP, Harlan JE, Yoon HS et al. X-ray and NMR structure of human Bcl-xL, an inhibitor of programmed cell death. Nature 1996; 381: 335-341.

50. Suzuki M, Youle RJ, Tjandra N. Structure of Bax: coregulation of dimer formation and intracellular localization. Cell 2000; 103: 645-654.

51. Chou JJ, Li H, Salvesen GS, Yuan J, Wagner G. Solution structure of BID, an intracellular amplifier of apoptotic signaling. Cell 1999; 96: 615-624.

52. McDonnell JM, Fushman D, Milliman CL, Korsmeyer SJ, Cowburn D. Solution structure of the proapoptotic molecule BID: a structural basis for apoptotic agonists and antagonists. Cell 1999; 96: 625-634.

53. Fesik SW. Insights into programmed cell death through structural biology. Cell 2000; 103 273-282.

54. Petros AM, Olejniczak ET, Fesik SW. Structural biology of the Bcl-2 family of proteins Biochim Biophys Acta 2004; 1644: 83-94.

55. Minn AJ, Velez P, Schendel SL, Liang H, Muchmore SW, Fesik SW et al. Bcl-x(L) forms an ion channel in synthetic lipid membranes. Nature 1997; 385: 353-357.

56. Sobko AA, Kotova EA, Antonenko YN, Zakharov SD, Cramer WA. Effect of lipids with different spontaneous curvature on the channel activity of colicin E1: evidence in favor of a toroidal pore. FEBS Lett 2004; 576: 205-210.

57. Annis MG, Soucie EL, Dlugosz PJ, Cruz-Aguado JA, Penn LZ, Leber B et al. Bax forms multispanning monomers that oligomerize to permeabilize membranes during apoptosis. EMBO J 2005; 24: 2096-2103.

58. Sattler M, Liang H, Nettesheim D, Meadows RP, Harlan JE, Eberstadt M et al. Structure of Bcl-xL-Bak peptide complex: recognition between regulators of apoptosis. Science 1997; 275: 983-986.

59. Dai H, Smith A, Meng XW, Schneider PA, Pang YP, Kaufmann SH. Transient binding of an activator $\mathrm{BH} 3$ domain to the Bak $\mathrm{BH}$-binding groove initiates Bak oligomerization. J Cell Biol 2011; 194: 39-48.

60. Czabotar PE, Westphal D, Dewson G, Ma S, Hockings C, Fairlie WD et al. Bax crystal structures reveal how $\mathrm{BH} 3$ domains activate $\mathrm{Bax}$ and nucleate its oligomerization to induce apoptosis. Cell 2013; 152: 519-531.

61. O'Neill JW, Manion MK, Maguire B, Hockenbery DM. BCL-XL dimerization by three-dimensional domain swapping. J Mol Biol 2006; 356: 367-381.

62. Denisov AY, Sprules T, Fraser J, Kozlov G, Gehring K. Heat-induced dimerization of BCL-XL through alpha-helix swapping. Biochemistry 2007; 46: 734-740.

63. Walensky LD. Direct BAKtivation. Nat Struct Mol Biol 2013; 20: 536-538.

64. Zhou HX, Cross TA. Influences of membrane mimetic environments on membrane protein structures. Annu Rev Biophys 2013; 42: 361-392.

65. Dewson G, Kratina T, Sim HW, Puthalakath H, Adams JM, Colman PM et al. To trigger apoptosis, Bak exposes its $\mathrm{BH} 3$ domain and homodimerizes via $\mathrm{BH} 3$ :groove interactions. Mol Cell 2008; 30: 369-380.

66. Bleicken S, Classen M, Padmavathi PV, Ishikawa T, Zeth K, Steinhoff HJ et al. Molecular details of Bax activation, oligomerization, and membrane insertion. J Biol Chem 2010 285: 6636-6647. 
67. Zhang Z, Zhu W, Lapolla SM, Miao Y, Shao Y, Falcone M et al. Bax forms an oligomer via separate, yet interdependent, surfaces. J Biol Chem 2010; 285: 17614-17627.

68. Dewson G, Ma S, Frederick P, Hockings C, Tan I, Kratina T et al. Bax dimerizes via a symmetric BH3:groove interface during apoptosis. Cell Death Differ 2012; 19: 661-670.

69. Ren D, Tu HC, Kim H, Wang GX, Bean GR, Takeuchi $O$ et al. BID, BIM, and PUMA are essential for activation of the BAX- and BAK-dependent cell death program. Science 2010; 330: 1390-1393

70. Gavathiotis E, Walensky LD. Tracking BAX once its trigger is pulled. Cell Cycle 2011; 10: 868-870.

71. Oltersdorf T, Elmore SW, Shoemaker AR, Armstrong RC, Augeri DJ, Belli BA et al. An inhibitor of Bcl-2 family proteins induces regression of solid tumours. Nature 2005; 435 677-681.

72. Tse C, Shoemaker AR, Adickes J, Anderson MG, Chen J, Jin S et al. ABT-263: a potent and orally bioavailable Bcl-2 family inhibitor. Cancer Res 2008; 68: 3421-3428.

73. Losonczi JA, Olejniczak ET, Betz SF, Harlan JE, Mack J, Fesik SW. NMR studies of the anti-apoptotic protein Bcl-xL in micelles. Biochemistry 2000; 39 11024-11033.

74. Franzin CM, Choi J, Zhai D, Reed JC, Marassi FM. Structural studies of apoptosis and ion transport regulatory proteins in membranes. Magn Reson Chem 2004; 42: 172-179.

75. Gong XM, Choi J, Franzin CM, Zhai D, Reed JC, Marassi FM. Conformation of membrane-associated proapoptotic tBid. J Biol Chem 2004; 279: 28954-28960.

76. Malia TJ, Wagner G. NMR structural investigation of the mitochondrial outer membrane protein VDAC and its interaction with antiapoptotic Bcl-xL. Biochemistry 2007; 46 514-525.

77. Denisov AY, Chen G, Sprules T, Moldoveanu T, Beauparlant P, Gehring K. Structural model of the BCL-W-BID peptide complex and its interactions with phospholipid micelles. Biochemistry 2006; 45: 2250-2256.

78. Xu XP, Zhai D, Kim E, Swift M, Reed JC, Volkmann N et al. Three-dimensional Structure of Bax-mediated Pores in Membrane Bilayers. Cell Death Dis 2013; 4: e683.

79. Oh KJ, Barbuto S, Meyer N, Kim RS, Collier RJ, Korsmeyer SJ. Conformational changes in BID, a pro-apoptotic BCL-2 family member, upon membrane binding. A site-directed spin labeling study. J Biol Chem 2005; 280: 753-767.

80. Epand RF, Martinou JC, Montessuit S, Epand RM. Transbilayer lipid diffusion promoted by Bax: implications for apoptosis. Biochemistry 2003; 42: 14576-14582.

81. Garcia-Saez AJ, Coraiola M, Serra MD, Mingarro I, Muller P, Salgado J. Peptides corresponding to helices 5 and 6 of Bax can independently form large lipid pores. FEBS 2006; 273: 971-981.

82. Qian S, Wang W, Yang L, Huang HW. Structure of transmembrane pore induced by Bax-derived peptide: evidence for lipidic pores. Proc Natl Acad Sci USA 2008; 105 17379-17383

83. Fuertes G, Garcia-Saez AJ, Esteban-Martin S, Gimenez D, Sanchez-Munoz OL, Schwille $\mathrm{P}$ et al. Pores formed by Baxalpha5 relax to a smaller size and keep at equilibrium. Biophys J 2010; 99: 2917-2925.

84. Bogner C, Leber B, Andrews DW. Apoptosis: embedded in membranes. Curr Opin Cell Biol 2010; 22: 845-851.

85. Cross TA, Sharma M, Yi M, Zhou HX. Influence of solubilizing environments on membrane protein structures. Trends Biochem Sci 2011; 36: 117-125

86. Katayama H, Wang J, Tama F, Chollet L, Gogol EP, Collier RJ et al. Three-dimensional structure of the anthrax toxin pore inserted into lipid nanodiscs and lipid vesicles. Proc Natl Acad Sci USA 2010; 107: 3453-3457.

87. Ye F, Hu G, Taylor D, Ratnikov B, Bobkov AA, McLean MA et al. Recreation of the terminal events in physiological integrin activation. J Cell Biol 2010; 188: 157-173.

88. Shi L, Shen QT, Kiel A, Wang J, Wang HW, Melia TJ et al. SNARE proteins: one to fuse and three to keep the nascent fusion pore open. Science 2012; 335: 1355-1359.

89. Parker MW, Tucker AD, Tsernoglou D, Pattus F. Insights into membrane insertion based on studies of colicins. Trends Biochem Sci 1990; 15: 126-129.

90. Song HY, Cohen FS, Cramer WA. Membrane topography of ColE1 gene products: the hydrophobic anchor of the colicin E1 channel is a helical hairpin. J Bacteriol 1991; 173 2927-2934.

91. Shin YK, Levinthal C, Levinthal F, Hubbell WL. Colicin E1 binding to membranes: time-resolved studies of spin-labeled mutants. Science 1993; 259: 960-963.
92. Schafer B, Quispe J, Choudhary V, Chipuk JE, Ajero TG, Du H et al. Mitochondrial outer membrane proteins assist Bid in Bax-mediated lipidic pore formation. Mol Biol Cell 2009; 20: 2276-2285

93. Pang X, Moussa SH, Targy NM, Bose JL, George NM, Gries C et al. Active Bax and Bak are functional holins. Genes Dev 2011; 25: 2278-2290.

94. Terrones O, Antonsson B, Yamaguchi H, Wang HG, Liu J, Lee RM et al. Lipidic pore formation by the concerted action of proapoptotic BAX and tBID. J Biol Chem 2004; 279: 30081-30091.

95. Kushnareva $Y$, Andreyev AY, Kuwana T, Newmeyer DD. Bax activation initiates the assembly of a multimeric catalyst that facilitates Bax pore formation in mitochondrial outer membranes. PLOS Biol 2012; 10: e1001394.

96. Campelo F, Fabrikant G, McMahon HT, Kozlov MM. Modeling membrane shaping by proteins: focus on EHD2 and N-BAR domains. FEBS Lett 2010; 584: 1830-1839.

97. Kozlov MM, McMahon HT, Chernomordik LV. Protein-driven membrane stresses in fusion and fission. Trends Biochem Sci 2010; 35: 699-706

98. McMahon HT, Kozlov MM, Martens S. Membrane curvature in synaptic vesicle fusion and beyond. Cell 2010; 140: 601-605.

99. Montessuit S, Somasekharan SP, Terrones O, Lucken-Ardjomande S, Herzig S, Schwarzenbacher $\mathrm{R}$ et al. Membrane remodeling induced by the dynamin-related protein Drp1 stimulates Bax oligomerization. Cell 2010; 142: 889-901.

100. Landeta O, Landajuela A, Gil D, Taneva S, Di Primo C, Sot B et al. Reconstitution of proapoptotic BAK function in liposomes reveals a dual role for mitochondrial lipids in the BAK-driven membrane permeabilization process. J Biol Chem 2011; 286: 8213-8230.

101. Martinez-Caballero S, Dejean LM, Kinnally MS, Oh KJ, Mannella CA, Kinnally KW. Assembly of the mitochondrial apoptosis-induced channel, MAC. J Biol Chem 2009; 284: 12235-12245.

102. Lee YJ, Jeong SY, Karbowski M, Smith CL, Youle RJ. Roles of the mammalian mitochondrial fission and fusion mediators Fis1, Drp1, and Opa1 in apoptosis. Mol Biol Cell 2004; 15: 5001-5011.

103. Takahashi Y, Karbowski M, Yamaguchi H, Kazi A, Wu J, Sebti SM et al. Loss of Bif-1 suppresses Bax/Bak conformational change and mitochondrial apoptosis. Mol Cell Biol 2005; 25: 9369-9382.

104. Etxebarria A, Terrones O, Yamaguchi H, Landajuela A, Landeta O, Antonsson B et al. Endophilin B1/Bif-1 stimulates BAX activation independently from its capacity to produce large scale membrane morphological rearrangements. J Biol Chem 2009; 284 : 4200-4212.

105. Cassidy-Stone A, Chipuk JE, Ingerman E, Song C, Yoo C, Kuwana T et al. Chemical inhibition of the mitochondrial division dynamin reveals its role in Bax/Bak-dependent mitochondrial outer membrane permeabilization. Dev Cell 2008; 14: 193-204.

106. Garcia-Saez AJ, Ries J, Orzaez M, Perez-Paya E, Schwille P. Membrane promotes tBID interaction with $\mathrm{BCL}(\mathrm{XL})$. Nat Struct Mol Biol 2009; 16: 1178-1185.

107. Denisov AY, Madiraju MS, Chen G, Khadir A, Beauparlant P, Attardo G et al. Solution structure of human $\mathrm{BCL}$-w: modulation of ligand binding by the C-terminal helix. J Biol Chem 2003; 278: 21124-21128.

108. Hinds MG, Lackmann M, Skea GL, Harrison PJ, Huang DC, Day CL. The structure of $\mathrm{Bcl}-\mathrm{w}$ reveals a role for the $\mathrm{C}$-terminal residues in modulating biological activity. EMBO $\mathrm{J}$ 2003; 22: 1497-1507.

109. Anfinsen CB. Principles that govern the folding of protein chains. Science 1973; 181: 223-230.

110. Murphy KM, Streips UN, Lock RB. Bax membrane insertion during Fas(CD95)-induced apoptosis precedes cytochrome c release and is inhibited by Bcl-2. Oncogene 1999; 18: 5991-5999.

111. Murphy KM, Ranganathan V, Farnsworth ML, Kavallaris M, Lock RB. Bcl-2 inhibits Bax translocation from cytosol to mitochondria during drug-induced apoptosis of human tumor cells. Cell Death Differ 2000; 7: 102-111.

112. Murphy KM, Streips UN, Lock RB. Bcl-2 inhibits a Fas-induced conformational change in the Bax N terminus and Bax mitochondrial translocation. $J$ Biol Chem 2000; 275: 17225-17228.

113. Billen LP, Kokoski CL, Lovell JF, Leber B, Andrews DW. Bcl-XL inhibits membrane permeabilization by competing with Bax. PLoS Biol 2008; 6: e147. 